\title{
Otimização da estabilidade de um gel fitoterápico usando Box-Behnken Design e Metodologia de Superfície de Resposta
}

\author{
Optimization of the stability of an herbal gel using Box-Behnken Design and Response Surface \\ Methodology \\ Optimizacion de la estabilidade de um gel de herbas mediante el diseño de Box-Behnken y la \\ Metodologia de Superfície de Respuesta
}

\section{Resumo}

A otimização de experimentos é um processo de reduz tempo, solventes, uso de equipamentos e melhora os resultados a partir de análise fatorial. A produção de géis fitoterápicos necessita de avaliação de estabilidade para poder ser utilizada de forma segura. Sendo assim, este estudo apresenta como objetivo otimizar por meio da análise fatorial do tipo Box-Behnken a produção de uma formulação de gel fitoterápico utilizando como variáveis dependentes os testes de densidade, espalhabilidade e $\mathrm{pH}$ obtidos durante a análise de estabilidade preliminar. As variáveis dependentes foram os percentuais do polímero de carbopol $940^{\circledR}$, de óleo de manga e do extrato de chá verde. Após a execução do fatorial, foram desenvolvidas 13 formulações em triplicatas, submetidas a centrifugação e 6 ciclos de estresse térmicos, no qual demonstraram caraterísticas físico-químicas aceitáveis, em seguida, observou-se utilizando gráficos de superfície de resposta baixos níveis de aderência dos ativos na formulação quando haviam altos índices de densidade e espalhamento. O ponto ótimo da formulação foi composto pelos níveis polímero -1 , extrato -1 e óleo +1 , com desejabilidade 0,67. Este ponto se mostrou reprodutível na prática. Portanto, sugere-se que a análise fatorial é uma ferramenta importante para a otimização e garantia de formulações fitoterápicas de qualidade e estáveis.

Palavras-chave: Fitoterapia; Estabilidade de cosméticos; Controle de qualidade; Otimização.

\begin{abstract}
The optimization of experiments is a process of reducing time, solvents, use of equipment and improves results from factor analysis. The production of herbal gels requires stability assessment in order to be used safely. Thus, this study aims to optimize the production of a herbal gel formulation using as dependent variables the density, spreadability and $\mathrm{pH}$ tests obtained during the preliminary stability analysis as dependent variables. The dependent variables were the percentages of carbopol polymer $940^{\circledR}$, mango oil and green tea extract. After the performance of the factorial, 13 formulations were developed in triplicates, submitted to centrifugation and 6 cycles of thermal stress, in which they demonstrated acceptable physical-chemical characteristics, then it was observed using surface charts of response
\end{abstract}


surface low levels of adhesion of the assets in the formulation when there were high density and scattering indexes. The optimum point of the formulation was composed of polymer -1 , extract -1 and oil +1 levels, with desirability 0.67. This point proved to be reproducible in practice. Therefore, it is suggested that factor analysis is an important tool for the optimization and guarantee of quality and stable herbal formulations.

Keywords: Phytotherapy; Cosmetic stability; Quality control; Optimization.

\section{Resumen}

La optimización de experimentos es un proceso de reducción de tiempo, disolventes, uso de equipos y mejora los resultados del análisis factorial. La producción de geles a base de hierbas requiere una evaluación de la estabilidad para ser utilizados de manera segura. Por lo tanto, este estudio tiene como objetivo optimizar la producción de una formulación de gel a base de hierbas utilizando como variables dependientes las pruebas de densidad, esparcibilidad y $\mathrm{pH}$ obtenidas durante el análisis preliminar de estabilidad como variables dependientes. Las variables dependientes fueron los porcentajes de polímero de carbopol $940^{\circledR}$, aceite de mango y extracto de té verde. Tras el desempeño del factorial, se desarrollaron 13 formulaciones en triplicados, sometidas a centrifugación y 6 ciclos de estrés térmico, en los que demostraron características físico-químicas aceptables, luego se observaron utilizando tablas superficiales de respuesta superficiales bajos niveles de adhesión de los activos en la formulación cuando había índices de alta densidad y dispersión. El punto óptimo de la formulación estuvo compuesto por polímero -1 , extracto -1 y aceite +1 niveles, con deseabilidad 0,67. Este punto demostró ser reproducible en la práctica. Por lo tanto, se sugiere que el análisis factorial es una herramienta importante para la optimización y garantía de formulaciones herbales de calidad y estables.

Palabras clave: Fitoterapia; Estabilidad cosmética; Control de calidad; Optimización.

\section{Introdução}

A utilização de ativos de origem vegetal, provenientes da flora brasileira aplicados à estética, visando traços mais jovens, tem movimentado o mercado e a pesquisa para o desenvolvimento de novas formulações cosméticas que apresentem segurança, eficácia e estabilidade confiável (Lima, et al., 2021). Dentre as ações terapêuticas, a atividade antioxidante tem destaque como proposta inovadora para esses produtos. As reações oxidativas são desencadeadas através do processo respiratório celular, onde as células aeróbicas levam à formação de radicais livres. Esses íons instáveis são um dos responsáveis por desenvolver muitas doenças, tais como: inflamações, tumores, doenças neurológicas e cardiovasculares, assim como aceleram o processo de envelhecimento da pele (Deus, et al., 2019).

A Mangifera indica, conhecida como manga, é uma fruta com alto teor vitamínico, principalmente vitamina A e C, além de apresentar uma rica fonte de carotenóides totais e compostos polifenóis (Santos, et al., 2021). Além da manga, outra planta medicinal promissora é a Camellia sinensi, popularmente denominada como chá verde, tem origem indiana, suas folhas secas são extraídas para produção de extratos e tinturas. Dentre suas propriedades bioativas destacam-se hepatoprotetora, antibacteriana, antialérgica, antineoplásicas, antioxidante e anti-inflamatória (Vilar, et al., 2019). Na área cosmética, o extrato de chá verde é, principalmente, utilizado na prevenção e reparo cutâneos. Sabe-se que seus metabólitos secundários atuam inibindo a enzima lipoxigenase e neutralizando as espécies reativas de oxigênio. Além disso, outra atividade de suma importância é a capacidade de inibir a enzima colagenase, justificando o uso dos seus extratos em formulações cosméticas (Zago \& Franzini, 2021; Martins, et al., 2021).

Desta forma, torna-se comum a inserção de antioxidantes não-enzimáticos, ou seja, aqueles não produzidos pelo organismo em formulações cosméticas, empregando em diversas formas farmacêuticas, no qual sua escolha dependerá da sua área de aplicação onde será administrado e da capacidade do cosmético em acondicionar e transferir essas substâncias para o organismo (Peçanha, et al., 2018).

O gel é uma forma farmacêutica semissólida que possui uma matriz polimérica que intumesce quando está em contato com um solvente. Dentre suas vantagens de utilização, destacam-se o baixo custo de produção, pureza e homogeneidade (Silva, 2021). Ademais, neste é possível veicular extratos e óleos vegetais (Almeida, et al., 2020). A fim de garantir qualidade e segurança das formulações cosméticas, estas, antes de ser disponibilizada ao consumidor, devem serem testadas e avaliadas para, assim, afirmar os requisitos básicos para utilização do produto. Nesse sentido, a Agência Nacional de Vigilância 
Sanitária, por meio da Gerência-Geral de Cosméticos, elaborou um guia para avaliar a estabilidade de produtos cosméticos. A análise de estabilidade dessas formulações cosméticas fornece informações que indicam as condições de necessários para sua fabricação até o término de sua validade, garantindo assim, que a mesma esteja em conformidade com o preconizado pelas autoridades sanitárias como comercialização (Anvisa, 2004; Ladeira, et al., 2021).

$\mathrm{O}$ estudo preliminar avalia a estabilidade de formulações durante a fase inicial do desenvolvimento do produto, a partir de variações que possam afetar a forma farmacêutica ou o ativo. Para a sua realização, as fórmulas propostas são submetidas a condições extremas de temperatura com o objetivo de acelerar possíveis reações de degradação entre seus componentes, e avaliar o surgimento de sinais de instabilidade e/ou características específicas de cada tipo de produto (Mazzo, et al., 2020; Pereira, et al., 2020).

Aliado ao teste de estabilidade preliminar, a utilização de métodos estatísticos para a observação dos estudos é de suma importância, pois, dessa forma, é possível selecionar com confiança o preparo da formulação (Jesus, et al., 2020). A otimização da produção da formulação a partir do planejamento fatorial do tipo Box-Behnken possibilita a percepção de adesão dos ativos, bem como da garantia da estabilidade do produto (Hoher, et al., 2020). Da mesma forma que possibilita reduzir o número de formulações produzidas, volume de reagentes utilizados e o tempo de execução do experimento garantindo produzir um produto em condições ótimas, com maior quantidade de ativos e estável (Gabbay Alves, et al., 2017). Portanto, este estudo tem como objetivo otimizar a produção de um gel fitoterápico contendo óleo de manga e extrato de chá verde, a partir de testes de qualidade realizados durante a etapa de estabilidade preliminar.

\section{Metodologia}

Para a síntese da formulação foram utilizados o óleo de manga (Moinho Central, Brasil), extrato de chá verde (Moinho Central, Brasil), carpopol 940 ${ }^{\circledR}$ (Cinética, Brasil), trietanolamina (Mapric, Brasil), metilparabeno (Êxodo científica, Brasil) e água destilada (Dinâmica, Brasil).

A otimização da formulação do gel foi realizada por meio de um planejamento fatorial do tipo Box-Behnken, utilizando software Design Expert ${ }^{\circledR}$ trial version (Gabbay Alves, et al., 2017). Para tal, foram utilizadas as variáveis independentes em três níveis $(-1,0,+1)$, no qual os percentuais utilizados do polímero de carbopol $940^{\circledR}$ foi de $0,5 \%$, do óleo de manga $1 \%$ e do extrato de chá verde $1 \%$ para nível -1 , no nível 0 foram aplicados polímero de carbopol $940^{\circledR}$ a $1,25 \%$, do óleo de manga $3 \%$ e do extrato de chá verde $3 \%$, e polímero de carbopol $940{ }^{\circledR} 2 \%$, do óleo de manga $5 \%$ e do extrato de chá verde $5 \%$ para o nível +1 , onde as variáveis dependentes foram a densidade, $\mathrm{pH}$ e espalhabilidade, totalizando 13 formulações (Tabela 1).

Tabela 1. Planejamento fatorial $2^{3}$ do tipo Box-Behnken.

\begin{tabular}{cccc}
\hline Formulações & Carbopol 940 $^{\circledR}$ & Óleo de manga & Extrato de chá verde \\
\hline 1 & +1 & +1 & +1 \\
2 & +1 & +1 & -1 \\
3 & 0 & 0 & 0 \\
4 & +1 & -1 & -1 \\
5 & 0 & 0 & 0 \\
6 & 0 & 0 & 0 \\
7 & 0 & 0 & 0 \\
8 & 0 & 0 & 0 \\
9 & +1 & -1 & +1 \\
10 & -1 & -1 & -1 \\
11 & -1 & +1 & -1 \\
12 & -1 & -1 & +1 \\
13 & -1 & +1 & +1 \\
\hline
\end{tabular}

Fonte: Autores. 


\section{Preparação das Formulações}

Durante a preparação das formulações foram pesados os sólidos e medidos os líquidos. Em um becker, foi adicionado água destilada, as partes sólidas e posto em uma manta aquecedora com agitação magnética. Após atingir $100^{\circ} \mathrm{C}$ e ocorrer a total solubilização do pó foi adicionado trietanolamina até a atingir pH 7,0 e, assim, alcançando a consistência de gel (Anvisa, 2019). Após esse procedimento foram incorporados os ativos de acordo com a Tabela 1.

\section{Teste de Centrifugação}

Antes de inciar o teste de estabilidade as amostras das formulações foram submetidas ao teste de centrifugação a 3.000 rpm durante 30 minutos. Qualquer evidência de instabilidade (separação de fase, oxidação ou mudança de coloração) a haveria a necessidade de reformulação. Esse procedimento resulta em um estresse termodinâmico, onde a presença de separação de fases, precipitação de substâncias e formação de caking (Mesquisa, et al., 2017).

\section{Ciclo Gelo-Degelo}

Sendo aprovadas após essa etapa de centrigugação, as fórmulas seguiram para o teste de estabilidade preliminar que consistiu em um ciclo de estresse térmico de gelo-degelo. O ciclo consiste na submição das fórmulações as seguintes condições de armazenamento: estufa $\left(40^{\circ} \mathrm{C} \pm 2^{\circ} \mathrm{C}\right)$ e geladeira $\left(-5^{\circ} \mathrm{C} \pm-2^{\circ} \mathrm{C}\right)$ (Anvisa, 2004; Garcia \& Pereira, 2020). Foram realizados 6 ciclos para análise desse estudo, em que cada ciclo era composto por 24 horas na temperatura da estufa e 24 horas na temperatura de geladeira, totalizando 48 horas, após o final de cada ciclo, as formulações foram submetidas as análises de controle de qualidade como avaliação organoléptica, espalhabilidade, densidade e pH. Para a avaliação dos parâmetros, o grupo controle foi deixado em temperatura ambiente, ou seja, sem sofrer a ação do estresse térmico (Anvisa, 2004; Garcia \& Pereira, 2020).

\section{Propriedades Organolépticas}

A cada ciclo foram avaliadas as propriedades organolépticas quanto ao aspecto, cor e odor, através de inspeção visual, tátil e olfativa (Anvisa, 2004; Garcia \& Pereira, 2020).

\section{Densidade Aparente}

A densidade foi calculada através do quociente entre a massa (g) pelo volume (L). Em uma balança analitica foi pesado a quantidade total do gel e anotado o valor da massa (Anvisa, 2004; Garcia \& Pereira, 2020).

\section{Espalhabilidade}

Para a determinação espalhabilidade, foram confeccionadas 3 peças. A primeira resulta em um disco de vidro transparente com diâmetro de $15 \mathrm{~cm}$ com um furo no centro de diâmetro de $1,2 \mathrm{~cm}$ (peça 1). A segunda era um disco de vidro transparente com diâmetro de $15 \mathrm{~cm}$ porém completamente íntegro (peça 2). E, por fim, foi feito uma peça de vidro transparente no formato quadrangular medindo $18 \mathrm{~cm}$ de largura por $18 \mathrm{~cm}$ de altura (peça 3). Para a realização do cálculo da espalhabilidade (Ei), foram pesados 0,3g das amostras, e as mesmas foram colocadas entre as peças 1 e 2, dispostas sobre a peça 3 na qual havia colado um papel milimetrado. A cada 3 minutos, foram adicionados sobre as peças pesos de 50, 150, 250 $3350 \mathrm{~g}$ e foi realizado a leitura do diâmetro da espalhabilidade (Silva, et al., 2019).

$$
E i=d^{2} \times \frac{\pi}{4}
$$

Onde: Ei = espalhabilidade da amostra para um determinado peso i $\left(\mathrm{mm}^{2}\right) ; \mathrm{d}=$ diâmetro médio $(\mathrm{mm})$. 
pH

Análise de pH foi realizada utilizando de tiras universais (KASVI, Brasil) com valores de 0 a 14 . As tiras eram mergulhadas nas formulações e observadas após 1 minuto (Anvisa, 2004; Garcia \& Pereira, 2020).

\section{Análise Estatística}

Após a realização dos testes de controle de qualidade durante o ciclo de gelo-degelo, esses resultados foram submetidos a análise de variância (ANOVA), na qual se aplica variáveis dependentes ou independentes para cada critério de estudo (Cordeiro, et al, 2018). Através deste teste, obtivemos o valor de F para determinação do grau de dispersão entre os dados da análise e valor de P, para determinação da significância dos achados do estudo, utilizando grau de confiança de 95\% e margem de erro de 5\%, desta forma considenrando valor de $\mathrm{p}=0,05$ (Cordeiro, et al, 2018).

\section{Resultados e Discussão}

Ao realizar o teste de triagem na centrifuga, todas as fórmulas mantiveram-se estáveis não apresentando nenhuma separação de fase, reação de oxidação ou mudanças organolépticas. Assim, todas as formulações foram dirigidas para a fase posterior, o teste preliminar. Durante os 6 ciclos de estresse térmico, macroscopicamente, foram observadas as condições organolépticas. Quando análisada a coloração do gel, este apresentou caráter translúcido e brilhoso, com coloração amarelo mostarda e odor característico do extrato de chá verde. Durante todos os ciclos essas características foram conservadas. Entretanto, o aspecto na formulação de concentração do polímero (nível 0), óleo (nível 0) e extrato (nível 0), foi verificado a presença de cristais. A presença desses cristais, podem ser resultantes do estresse térmico submetido durante o teste de gelodegelo. Isso sugere a não conformidade no processo de fabricação da forma farmacêutica, e/ou incompatibilidade física dos componentes da formulação (Anvisa, 2004).

Para a avaliação de controle de qualidade, a cada término do ciclo de estresse térmico, foram realizados os testes fisíco-químicos das amostras, os quais podem ser observados nos gráficos de superficie de resposta (Figura 1), em que foram mantidos o percentual fixo do polímero (nível 0). A Figura 1 mostra que a densidade e a espalhabilidade aumentam de forma diretamente proporcional ao percentual do extrato. Entretanto, o pH reduz quando se tem maior percentual de extrato. Ressaltase que os valores do óleo de manga adicionados não afetaram as variáveis dependentes, talvez isto possa estar relacionado com a afinidade da matriz do gel em ativos de mesma polaridade, como o extrato de chá verde, portanto a partir da interação entre ambos pode-se haver diferenças dos parâmetros físico-químicos.

Figura 1. Análise de superfície de resposta segundo o planejamento fatorial executado das variáveis dependentes em função do polímero 0: A) Densidade; B) Espalhabilidade; C) $\mathrm{pH}$.
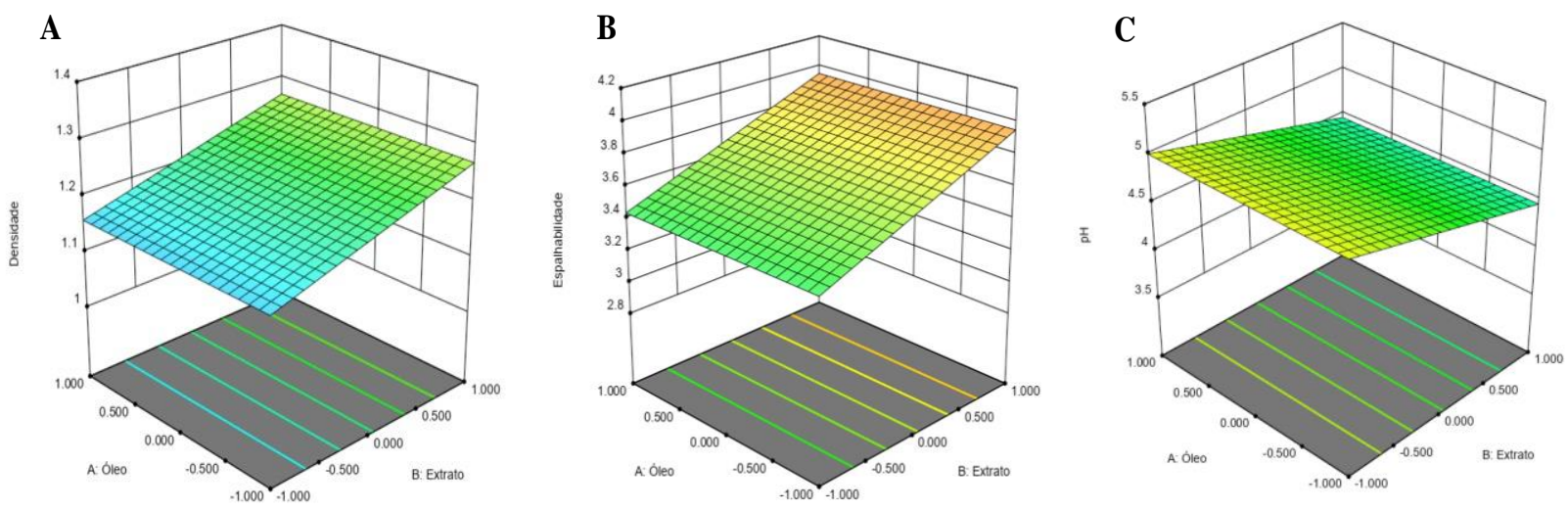

Fonte: Autores (2022). 
Santos, et al. (2021) relatam que no desenvolvimento de uma formulação fitocosmética, ao passo que a concentração de ativos adicionados aumentava a densidade reduzia. Esse fato pode ser explicado devido à diferença de polímero utilizado, assim como em função da polaridade dos ativos.

Já Costa, et al. (2021) pontuam que é muito comum a incompatibilidade entre formulação semissólida (gel) e ativos vegetais, principalmente pela interatividade do polímero com o extrato vegetal. A incompabilidade pode ser apresentada por características físicas onde há a alteração organoléptica da formulação, o que não foi observado na análise das formulações encontrando-se em coloração e odor adequados (Anvisa, 2004). A alteração de natureza química pode se dar pela incompatibilidade quanto ao $\mathrm{pH}$, reações de oxidação, hidrólise na formulação e/ou possíveis interações entre os componentes da formulação (Anvisa, 2004).

Apesar disso, os resultados obtidos para espalhabilidade confluíram com uma pesquisa, na qual observou que quando menor a viscosidade da formulação maior será sua espalhabilidade (Martins, et al., 2016). A análise desse parâmetro é um dado imprescindível para a formulação, visto que seu resultado demonstra informações importantes sobre a eficácia, uma vez que está diretamente relacionada com o aumento da absorção da forma farmacêutica semissólida (Santos, et al., 2021). Quanto ao valor do $\mathrm{pH}$, foi analisado que, a medida que o nível de óleo aumenta e o nível de extrato diminui, esse fator cresce. Esse dado coincidiu com um estudo que, também, observou uma tendência na elevação do pH em uma formulação que apresentava maior teor lípídico (Santos, et al., 2021). Costa (2016), discorre que para atingir a máxima espalhabilidade com o polímero carbopol, o pH deve estar em torno de 7, ou seja, neutro, no entanto são aceitáveis valores mais ácidos entre 5 a 6.

A Figura 2 evidencia as distribuições de omitização das formulações, nota-se que o grau de desejabilidade foi de 0,673 (Figura 2A), ou seja, é o ponto otimizado de acordo com a predileção previamente estipulada no programa. Para este ponto, as melhores condições ótimas para as variáveis independentes foram: percentual do gel de Carbopol -1, óleo de manga +1 e extrato de chá verde -1. Segundo esses, pode-se propor também as condições de densidade 1,13 (Figura 2B), espalhabilidade 3,43 (Figura 2C) e pH 5,00 (Figura 2D). As otimizações das variáveis dependentes e independentes foram calculadas pelo software Design Expert ${ }^{\circledR}$ com intervalo de confiança de $95 \%$.

Figura 2. Distribuição da otimização numérica quanto a: A) Desejabilidade; B) Densidade; C) Espalhabilidade; e D) pH.
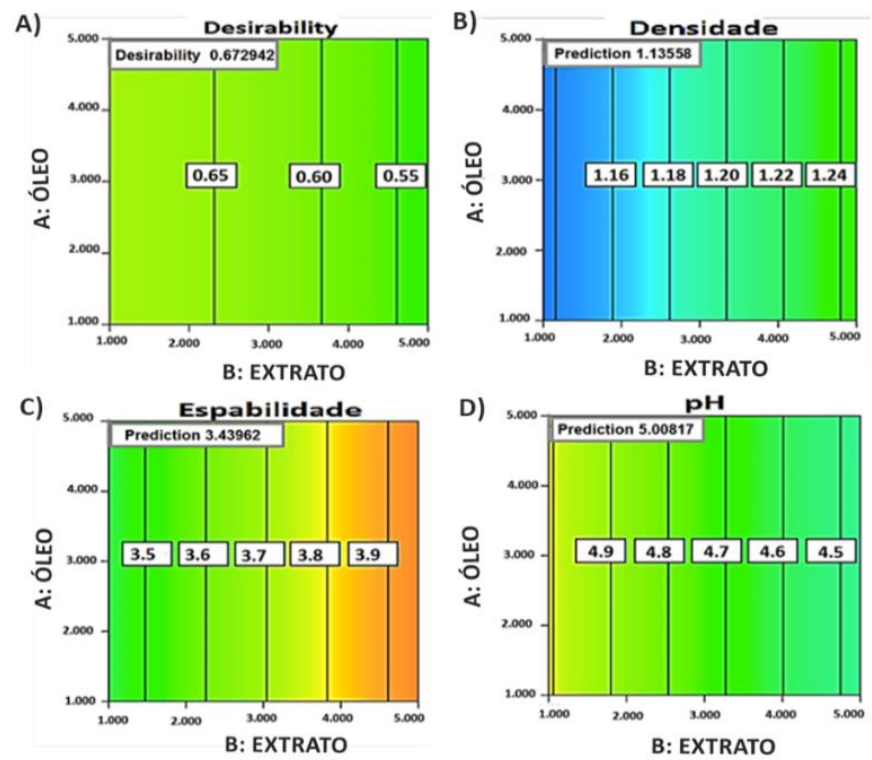

Fonte: Autores (2022). 
Diante de todos os valores otimizados, uma amostra do ponto ótimo foi reproduzida e foram testados em triplicata os aspectos organolépticos (translúcido e brilhoso), densidade $(1,27 \pm 0,07)$, espalhabilidade $(3,14 \pm 0,38)$ e $\mathrm{pH}(5,00 \pm 0,01)$. Assim, pode-se observar que os valores práticos foram muito próximos dos pontos estabelecidos pelo programa, sugerindo reprodutibilidade. A análise de variância (ANOVA), mostrou que o p-valor do método proposto foram para a densidade $(0,0417), \mathrm{pH}(0,0853)$ e espalhabilidade $(0,0397)$, demonstrando maior significância para densidade e espalhabilidade, ressaltando o valor-f que aponta medidas de dispersão de 4,44 e 5,44 respectivamente, como demonstra a Tabela 2.

Tabela 2. Análise de Variância (ANOVA).

\begin{tabular}{llrrrrr}
\hline Teste & & SS & DF & MS & F-value & p-value \\
\hline Densidade & Model & 0,0282 & 2 & 0,0141 & 4,44 & 0,0417 \\
& Residual & 0,0318 & 10 & 0,0032 & & \\
& Lack of fit & 0,0213 & 6 & 0,0036 & 1,35 & 0,4024 \\
& Total & 0,0813 & 18 & & & \\
\hline Espalhabilidade & Model & 0,5202 & 2 & 0,5202 & 5,44 & 0,0397 \\
& Residual & 1,05 & 11 & 0,0956 & & \\
& Lack of fit & 0,7498 & 7 & 0,1071 & 1,42 & 0,3866 \\
& Total & 2,32 & 20 & & & \\
\hline $\mathrm{pH}$ & Model & 0,5886 & 1 & 0,5886 & 3,57 & 0,0853 \\
& Residual & 1,81 & 11 & 0,1645 & & \\
& Lack of fit & 0,5059 & 7 & 0,0723 & 0,2214 & 0,9595 \\
& Total & 2,9045 & 19 & & & \\
\hline
\end{tabular}

SS: soma dos quadrados; DF: graus de liberdade; MS: quadrado médio; F-value: variável de dispersão; P-Value: valor de hipótese <0,05. Fonte: Autores (2022).

Assim, o ponto de otimização foi feito e as respostas se mantiveram dentro do intervalo de $95 \%$ de confiança estabelecido pelo programa. $\mathrm{Na}$ análise de densidade obteve-se dados satisfatórios $(\mathrm{p}=0,0417)$ podendo assim expressar um produto com baixos índices de aeração da formulação, no qual se mantiveram níveis de repetibilidade aceitáveis no teste da formulação $(F=4,44)$ (Alves, et al., 2021). Desta forma, sugere-se que este não foi o fator primordial na perda do concentrado dos ativos voláteis da formulação (Anvisa, 2004).

Ao analisar o perfil de espalhabilidade da formulação, demonstrou-se dados significativos ( $\mathrm{p}=0,0397)$, sugerindo que a formulação se mantém em condições de uso em diferentes aplicações de força e com diferentes níveis de concentração dos ativos, observado pela variação no valor de $\mathrm{F}(\mathrm{F}=5,44)$. Este dado corrobora com a literatura, uma vez que a espalhabilidade promove maior cobertura da formulação na superfície da pele, conferindo melhor eficiência ao produto, assim como consequentemente maior aceitabilidade pelo consumidor (Almeida, et al., 2020; Alves, et al., 2021).

Desta forma, visto que $\mathrm{pH}$ é um fator importante nos testes de cosméticos, para garantir a estabilidade e eficácia da formulação, observa-se que no gel testado da formulação não expressa dado satisfatório ( $\mathrm{p}=0,0853)$ para sua viabilização. Segundo Silva, et al. (2019), esta instabilidade pode se dar devido a variações decorrentes da incompatibilidade dos componentes presentes na formulação. Sugerindo este como o ponto causador da instabilidade, apesar do mesmo ter apresentado variação mínima quanto as suas testagens $(F=3,57)$, demonstrou-se que não houve grande variação entre as triplicatas (Silva et al, 2019).

Sendo assim, pontuou-se que a densidade e espalhabilidade das amostras testadas são efetivas para a aplicação dos extratos e óleos em formulações de géis, visto que se houve um valor satisfatório estatisticamente, corroborando com a variabilidade demonstrada nas análises de triplicata, constatado nos valores de $\mathrm{F}$ (Tabela 2). Porém, a formulação não se 
encontra em ajuste quanto ao $\mathrm{pH}$, sugerindo instabilidade do produto, menor prazo de vida útil da formulação e em alguns casos agrega risco na sua utilização.

\section{Considerações Finais}

A estabilidade preliminar mostrou que as 13 formulações atenderam às especificações de qualidade necessária, exceto nas concentrações de polímero (nível 0), óleo (nível 0) e extrato (nível 0) na qual houve cristalização. As formulações demonstraram-se efetivas nos testes de densidade e espalhabilidade, porém, em desacordo com os níveis de $\mathrm{pH}$, ocasionando um maior percentual de perda do ativo da formulação. Baseado em estudos, destacamos a importância da análise fatorial para a diminuição do número de experimentos, para assegurar as respostas em relação a estabilidade de formulações. Portanto, sugere-se que a otimização numérica produziu a formulação mais estável de forma preliminar acordo com as variáveis independentes nas concentrações de polímero (nível 0), óleo (nível 0) e extrato (nível 0) e das variáveis dependentes estudadas. Por fim, sugerimos a aplicação de estudos futuros para aplicação de testes de qualidade a fim de garantir a viabilização da formulação para aplicação, tais como: controle microbiológico, avaliação da comedogênicidade e testes de irritabilidade dérmica primária, acumulada e sensibilização.

\section{Agradecimentos}

Todos os autores agradecem a Universidade da Amazônia (UNAMA), campus Ananindeua, pelo suporte durante a realização deste estudo.

\section{Referências}

Almeida, L. C., Silva, D. C., Couto, B. S., Silva, K. O., \& Braz, W. R. (2020). Avaliação da qualidade de formulações magistrais fotoprotetoras géis-creme comercializadas na região centro oeste de Minas Gerais. Revista Conexão Ciência. 5 (3), 24-42. https://periodicos.uniformg.edu.br:21011/ojs/index.php/conexaociencia/index

Alves, P. H. P., Ugoline, B. C. A., \& Bastos, J. C. S. A. (2021). Avaliação da estabilidade preliminar de géis contendo extratos glicólicos de Barbatimão (Stryphnodendron barbatiman M.) e Hamamélis (Hamamelis virginiana L.). Ágora, Revista Científica da FaSar. 3 (1). https://www.fasar.com.br/wpcontent/uploads/2021/05/CEPIC-ARTIGO-AGORA-2021-PRISCILLA-E-BRUNO.pdf

Anvisa. (2004). Guia de Estabilidade de Produtos Cosméticos, Brasil: Editora Anvisa.

Anvisa. (2019). Farmacopéia Brasileira. (6a ed.), Brasil: Editora Anvisa.

Cordeiro, E. D. A., Melo, M. B., \& Fernandes, S. C.S. (2018). Um estudo sobre a utilização da ANOVA de uma via produção científica na área de psicologia. Meta: Avaliação, 10 (28), 139-153. 10.22347/2175-2753v10i28.1455

Costa, P.D. (2016). Desenvolvimento e validação do método analítico dos produtos intermediários e estudo de estabilidade preliminar de gel de Thuja occidentalis Linn. (Cupressaceae) para terapia antiviral (Dissertação de mestrado). Universidade Federal de Pernambuco - UFPE. Recife, Brasil.

Costa, R. S., Costa, R. M. R., \& Júnior, J. O. C. S. (2021). Comportamento reológico e atividade antimicrobiana de uma formulação tópica contendo extrato de Heliotropium indicum L. Research, Society and Development, 10 (5), 32310515068. doi:10.33448/rsd-v10i5.15068

Deus, V. L., Santos, A. P. C., Walker, J. F., Santana Neta, L. G., \& Souza, L. S. (2019). Compostos fenólicos em hortaliças cultivadas nos sistemas convencional e orgânico: uma revisão. Brazilian Journal of Health and Pharmacy. 1 (1), 70-84. 10.29327/226760.1.1-8

Gabbay Alves, T. V., Costa, R. S., Aliakbarian, B., Casazza, A. A., Perego, P., Júnior J. O. C. S., Costa, R. M. R., \& Converti, A. (2017). Microencapsulation of Theobroma cacao L. waste extract: optimization using response surface methodology. Journal Microencapsul. 34 (2),111-120. $10.1080 / 02652048.2017 .1296499$

Garcia, L. L. O., \& Pereira, T. A. (2020). Avaliação da estabilidade de fel creme contendo extrato de Solidago chilenses Meyen (Trabalho de conclusão de curso). Universidade de Uberaba. Uberaba, Minas Gerais (MG), Brasil.

Hoher, G. L., Cascaes, B. P., Rodrigues, L. J., \& Schneider, P. S. (2020). Análise dos parâmetros de um sistema de aquecimento de água via Box-Behnken. VIII Congresso Brasileiro de Energia Solar, Fortaleza, Brasil.

Jesus, J. F., Reis, I. M. A., \& Costa, S. C. C. (2020). Avaliação da estabilidade de pastilha gomosa contend extrato seco de Citrus aurantium. Brazilian Journal of Health Review. 3 (3), 34327-4350. doi:10.34119/bjhrv3n3-034 
Ladeira, G. D. A., Alves, A. S. A., Mota, B. S., Rosa, J. A., Gomes, M. G. R., Tavares, R. C., \& Xavier, Q. M. L. (2021). A importância dos estudos de préformulação na estabilidade dos produtos cosméticos. Revista Ibero-Americana de Humanidades, Ciências e Educação. 7 (12), 1074-1085. $10.51891 /$ rease.v7i12.3555

Lima, L R., Costa, J. R. L., Bena, M. G. P., Gomes, M. T. H. C. A B., Sousa, J. A. B., Bacelar, S. N. A., Paz, B. K. B., \& Mascarnhas, M. T. M. (2021). Cosméticos orgânicos: uma tendência crescente no mercado. Brazilian Journal of Development, 7 (1), 4322-4331. doi:10.34117/bjdv7n1-291

Martins, N., Roriz, C. L., Morales, P., Barros, L., \& Ferreira, I. C. F. R. (2016). Food colorants: Challenges, opportunities and current desires of agroindustries to ensure consumer expectations and regulatory practices. Trends in Food Science \& Technology, 52 (1), 1-15. 10.1016/j.tifs.2016.03.009

Martins, R. B., Seibel R., Ceolin, J., Junges, V. M., \& Gottlieb, M. G. V. (2021). Uso potencial do chá verde no tratamento complementar de morbidades e uso de medicamentos, associado ao envelhecimento: uma revisão. Editora Ciência Digital. Envelhecimento Humano Desafios Contemporâneos, 2 (27), 1-17. $10.37885 / 201202390$

Mazzo, J. S O., Guidi, A. C., Lima, C. B., Santos, R. S., Teston, A. P. M., Bruschi, M. L., Mello, J. C. P., \& Araújo, D. C. M. (2020). Desenvolvimento farmacotécnico de formulações de fotoprotetores FPS 30 e avaliação da estabilidade preliminar. Brazilian Journal of Development. 6 (8), 63696-63711. doi:10.34117/bjdv6n8-703

Mesquisa, S. S., Teixeira, C. M. L. L., \& Servulo, E. F. C. (2017). Carotenóides: Propriedades, Aplicação e Mercado. Revista Virtual de Química, 9 (2), $672-$ 688. $10.21577 / 1984-6835.20170040$

Peçanha, F., Trindade, G. O., Marino, P. A., \& Maldaner, G. (2018). Análise do teor de polifenóis em diferentes amostras de Camellia sinensis. Anais do 10 Salão Internacional de Ensino, Pesquisa e Extensão (SIEPE), Santana do Livramento, Brasil.

Pereira, G. C., Murat, S. C. M., Magalhães, B. S., Benevenuto, B. R., Silva, L. D., Ribeiro, R. S. G., \& Pereira, C. S. S. (2020). Avaliação da estabilidade de um xampu produzido com adição de óleo essencial de alecrim (Rosmarinus officinalis). Revista Teccen. 13 (1), 02-07. 10.21727/teccen.v13i1.2328

Santos, F. D. R. P., Belfort, M. G. S., Luceus, B. V., \& Chaves, J. A. (2021). Características físico-químicas de um sérum desenvolvido à base de óleo de Buriti (Mauritia flexuosa) para pele idosa. Revista de Enfermagem Atual In Derme, 95 (33), 1-15. 10.31011/reaid-2021-v.95-n.33-art.912

Silva, F. V. F., Santos, M. C., Neiva, L. D. B., Oliveira, M. A. C., Leal, B. S., Moreira, F. A. S., \& Sousa, J. P. S., (2019). Desenvolvimento e controle de qualidade de um gel-creme antiacneico a base do óleo da Copaifera officinalis L. (copaíba). Revista Eletrônica Acervo Saúde / Electronic Journal Collection Health. 30 (974), 1-10. 10.25248/reas.e974.2019

Silva, R. R. (2021). Estudo da estabilidade preliminar de formulações contendo extrato de maruré (Brosium acutifolium). (Trabalho de conclusão de curso). Universidade Federal do Amazônas - UFMA, Manaus, Brasil.

Silva, T. F., Bortolotto, J. W., Deuschle, R. A. N., Claudino, T. S., \& Deuschle, V. C. K. N. (2019). Desenvolvimento e estudo de estabilidade físico-química de formulações cosméticas antienvelhecimento. Revista Contexto e Saúde, 19 (36), 107-113. 10.21527/2176-7114.2019.36.107-113

Vilar, D. A., Vilar, M. S. A., Brandão, M. P. Anjos, C. J. F., \& Silva, A. E. (2019). Plantas Medicinais: Um guia prático. Instituto Federal de Sergipe. Aracaju: Brasil.

Zago, E. C. \& Franzini, C. M. (2021). Revisão Sistemática sobre a ação do licopeno perante ao envelhecimento cutâneo em nutricosméticos. Revista IberoAmericano de Humanidades, Ciências e Educação. 7 (9), 863-883. 10.51891/rease.v7i9.2286 